\title{
Editorial: Nutrient Interactions in Plants
}

\author{
Francisco Javier Romera ${ }^{1 *}$, Ping Lan ${ }^{2 *}$, Jorge Rodríguez-Celma ${ }^{3 *}$ and \\ Rafael Pérez-Vicente ${ }^{4 *}$
}

${ }^{1}$ Department of Agronomy-Universidad de Córdoba (DAUCO-María de Maeztu Unit of Excellence), Edificio Celestino Mutis (C-4), Campus de Excelencia Internacional Agroalimentario de Rabanales CeiA3, Universidad de Córdoba, Córdoba, Spain, ${ }^{2}$ State Key Laboratory of Soil and Sustainable Agriculture, Institute of Soil Science, Chinese Academy of Sciences, Nanjing, China, ${ }^{3}$ Plant Nutrition Department, Aula Dei Experimental Station, CSIC (Consejo Superior de Investigaciones Cientificas), Zaragoza, Spain, ${ }^{4}$ Department of Botany, Ecology and Plant Physiology, Edificio Celestino Mutis (C-4), Campus de Excelencia Internacional Agroalimentario de Rabanales CeiA3, Universidad de Córdoba, Córdoba, Spain

Keywords: crosstalk, mineral nutrition, nutrient, nutrient acquisition, nutrient deficiency responses, nutrient interactions

\section{Editorial on the Research Topic}

\section{Nutrient Interactions in Plants}

Plants, like other living organisms, require an assemblage of essential elements to synthesize their constituent compounds and for essential metabolic reactions. Besides carbon $(\mathrm{C})$, hydrogen $(\mathrm{H})$ and oxygen $(\mathrm{O})$, plants require 14 essential mineral elements such as nitrogen $(\mathrm{N})$, phosphorus $(\mathrm{P})$, potassium $(\mathrm{K})$, sulfur $(\mathrm{S})$, magnesium $(\mathrm{Mg})$, calcium $(\mathrm{Ca})$, zinc $(\mathrm{Zn})$, iron $(\mathrm{Fe})$, copper $(\mathrm{Cu})$, manganese (Mn), molybdenum (Mo), nickel (Ni), chlorine (Cl) and boron (B) (Marschner, 2012). Additionally, there are other mineral elements that are not essential for all plant species but that can be beneficial for some groups of plants, like sodium (Na; Maathuis, 2014) or silicon (Si; Tripathi et al., 2020). All these elements interact in a direct and/or indirect manner. In some cases, the deficiency or excess of one element can affect the uptake of other(s), thus conditioning their proper uptake and efficient utilization (Astolfi et al., 2021; Bernal and Kramer, 2021; Pavlovic et al., 2021; Yu et al.; Zhou et al.). For instance, S deficiency can limit Fe acquisition (Astolfi et al., 2021) while $\mathrm{P}$ deficiency can promote it (Figure 1; García et al.). On the other hand, P excess can diminish $\mathrm{Zn}$ acquisition (Yu et al.). In other cases, a scarce element, i.e., K, can be substituted by another element of similar characteristics, like $\mathrm{Na}$ (Mateus et al.).

This Research Topic updates recent results showing the interactions between different essential mineral nutrients, and also between essential and non-essential ones. It includes 5 reviews, 1 minireview, 1 perspective and 13 original research articles. Regarding the reviews; one is related to interactions between two essential elements, S and Fe (Astolfi et al., 2021); two are related to interactions between macro- and micro-nutrients (Fan et al., 2021; Kumar et al., 2021); and the other two are dedicated to interactions between beneficial elements, Si and Se, and essential ones (Pavlovic et al., 2021; Zhou et al.). The minireview deals with the interaction between $\mathrm{N}$ and $\mathrm{P}$ in the development of root nodules and cluster roots (Pueyo et al., 2021). The perspective article is devoted to describing new approaches based on computational analysis to predict interactions between proteins related to different elements (Di Silvestre et al.). Finally, within the 13 original research articles; eight of them are about the interactions between two or three elements, including non-essential ones, such as Fe-Cu, Si-Fe, Fe-P-S, S-N, Fe-Zn, K-Na, or P-Zn (Bernal and Kramer, 2021; Jian et al., 2021; Kakei et al., 2021; Li et al., 2021; Chaiwong et al.; García et al.; Suman et al.; Yu et al.); four of them are about the interactions among many nutrients, such as ionomemacronutrients, ionome-micronutrients, ionome-N (Courbet et al.; D'Oria et al.; Zhang C. et al.; Zhang J. et al.); and one is devoted to the substitution of $\mathrm{K}$ by $\mathrm{Na}$ (Mateus et al.). 


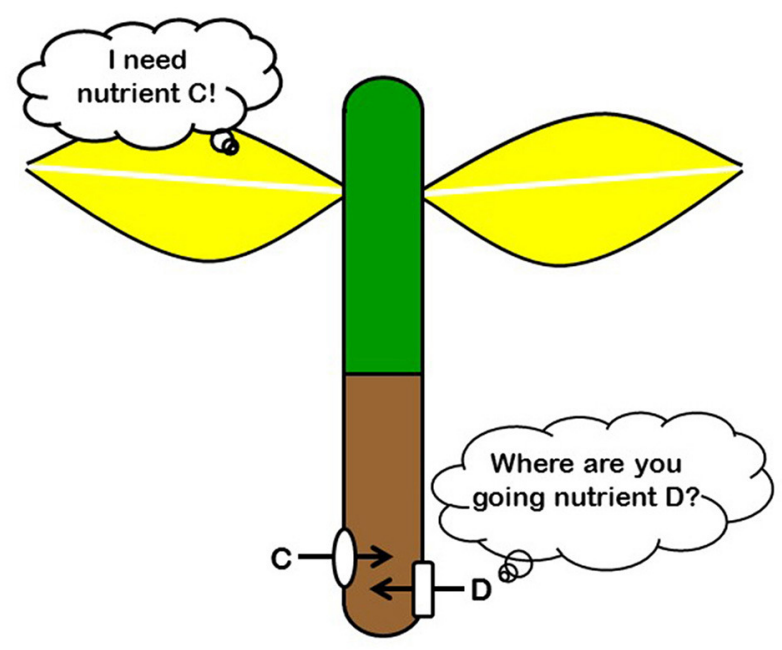

FIGURE 1 | The responses to the deficiency of a particular nutrient can promote the acquisition of such a nutrient but also the acquisition of other nutrients.

The articles included in this Research Topic reflect indirect interactions between nutrients, such as those simultaneously analyzing many nutrients (Courbet et al.; D'Oria et al.; Zhang C. et al.; Zhang J. et al.), and also direct interactions, like those studying the interplay between 2 and 3 elements (Bernal and Kramer, 2021; Jian et al., 2021; Kakei et al., 2021; Li et al., 2021; Chaiwong et al.; García et al.; Mateus et al.; Suman et al.; Yu et al.). The depicted interactions occur at different steps of nutrient acquisition and translocation inside the plant. For instance, $\mathrm{P}$ deficiency, through organic acid release and rhizosphere acidification, can promote the mobilization of other nutrients, like Fe or $\mathrm{Zn}$ (Pueyo et al., 2021). In the same way, $\mathrm{Si}$ application can promote $\mathrm{N}$ and $\mathrm{P}$ acquisition by upregulating nitrate and phosphate transporters (Pavlovic et al., 2021). New interactions are described in this Research Topic, like the uptake of vanadium mediated by sulfate transporters whose expression was stimulated during $\mathrm{S}$ deprivation (Courbet et al.). In relation to the translocation of some elements, like $\mathrm{Fe}, \mathrm{Cu}$ and $\mathrm{Mn}, \mathrm{S}$ deficiency can negatively affect it by limiting the biosynthesis of nicotianamine, a chelating agent linked to this process (Astolfi et al., 2021).

In this Research Topic, mechanisms underlying the observed interactions are proposed. Two elements can interact because they share similar chemical properties, like $\mathrm{K}$ and $\mathrm{Na}$ (Mateus et al.). One element can participate in compounds or proteins involved in key processes related to others [i.e., S-containing metabolites participate in the synthesis of ethylene and phytosiderophores, which are in turn implicated in Fe uptake (Astolfi et al., 2021); a multicopper oxidase participates in $\mathrm{Fe}$ translocation (Bernal and Kramer, 2021)]. The participation of different elements in the same compounds (i.e., $\mathrm{N}$ and $\mathrm{S}$ in methionine and cysteine; $\mathrm{Fe}$ and $\mathrm{S}$ in $\mathrm{Fe}-\mathrm{S}$ clusters) can also cause their interactions (Astolfi et al., 2021). Finally, the participation of the same phytohormones, signaling molecules (nitric oxide, miRNAs, peptides and others), and transcription factors in the homeostasis of different elements can explain the interactions between them (Astolfi et al., 2021; Bernal and Kramer, 2021; García et al., 2021; Kumar et al., 2021; Pueyo et al., 2021; Chaiwong et al.; García et al.; Mateus et al.). For instance, ethylene and nitric oxide upregulate both $\mathrm{P}$ - and $\mathrm{Fe}$-acquisition genes in such a way that the deficiency of either of them, that stimulate the production of ethylene and nitric oxide, promote the acquisition of the other one (García et al.).

Interactions between nutrients can have many different consequences, depending on them being essential or beneficial, and on other factors. In this sense, it is important to point out that the interactions between nutrients greatly depend on the severity of the nutrient deficiency or excess (Astolfi et al., 2021). For instance, Si upregulates nitrate and phosphate transporters when plants are grown under limiting $\mathrm{N}$ and $\mathrm{P}$ conditions but downregulates them when grown under sufficient $\mathrm{N}$ and $\mathrm{P}$ conditions (Pavlovic et al., 2021). Besides nutrition, interactions between nutrients can affect other processes, like the accumulation of secondary metabolites (Jian et al., 2021). It is important to note the interest of the interactions between essential and non-essential elements, since non-essential elements, like $\mathrm{Na}$, can partially substitute for essential ones, like $\mathrm{K}$ (Mateus et al.). Additionally, non-essential elements, like Si, can affect the homeostasis of essential elements (Pavlovic et al., 2021), and vice versa (Chaiwong et al.; Zhou et al.). A better knowledge of such interactions could aid in the improvement of some nutritional disorders and/or in the biofortification of some essential elements for humans and animals, like Se (Zhou et al.).

In conclusion, the better understanding of the interactions between elements (essential and non-essential) could lead to more rational fertilization practices, preventing interactions that could contribute to an unbalanced mineral nutrition of plants. This knowledge is also necessary to obtain more efficient genotypes in the acquisition of the different nutrients.

\section{AUTHOR CONTRIBUTIONS}

All authors listed have made a substantial, direct, and intellectual contribution to the work and approved it for publication.

\section{FUNDING}

PL was funded by the National Natural Science Foundation of China (32070279). FJR and RPV were funded by the Spanish Ministry of Science and Innovation (RTI2018-097935-B-I00), the Spanish State Research Agency, through the Severo Ochoa and María de Maeztu Program for Centers and Units of Excellence in R\&D (Ref. CEX2019-000968-M), and the 'Junta de Andalucía' (Research Groups AGR115 and BIO159). 


\section{REFERENCES}

Astolfi, S., Celletti, S., Vigani, G., Mimmo, T., and Cesco, S. (2021). Interaction between sulfur and iron in plants. Front. Plant Sci. 12:670308. doi: 10.3389/fpls.2021.670308

Bernal, M. I., and Kramer, U. (2021). Involvement of Arabidopsis multicopper oxidase-encoding LACCASE12 in root-to-shoot iron partitioning: a novel example of copper-iron crosstalk. Front. Plant Sci. 12:688318. doi: 10.3389/fpls.2021.688318

Fan, X., Zhou, X., Chen, H., Tang, M., and Xie, X. (2021). Cross-talks between macro- and micro-nutrient uptake and signalling in plants. Front. Plant Sci. 12:663477. doi: 10.3389/fpls.2021.663477

García, M. J., Lucena, C., and Romera, F. J. (2021). Ethylene and nitric oxide involvement in the regulation of $\mathrm{Fe}$ and $\mathrm{P}$ deficiency responses in dicotyledonous plants. Int. J. Mol. Sci. 22:4904. doi: 10.3390/ijms22094904

Jian, S. F., Huang, X. J., Yang, X. N., Zhong, C., and Miao, J. H. (2021). Sulfur regulates the trade-off between growth and andrographolide accumulation via nitrogen metabolism in Andrographis paniculata. Front. Plant Sci. 12:687954. doi: $10.3389 /$ fpls.2021.687954

Kakei, Y., Masuda, H., Nishizawa, N. K., Hattori, H., and Aung, M. S. (2021). Elucidation of novel cis-regulatory elements and promoter structures involved in iron excess response mechanisms in rice using a bioinformatics approach. Front. Plant Sci. 12:660303. doi: 10.3389/fpls.2021.660303

Kumar, S., Kumar, S., and Mohapatra, T. (2021). Interaction between macro- and micro-nutrients in plants. Front. Plant Sci. 12:665583. doi: 10.3389/fpls.2021.665583

Li, T., Li, Y., Sun, Z., Xi, X., Sha, G., Ma, C., et al. (2021). Resveratrol alleviates the $\mathrm{KCl}$ salinity stress of Malus hupehensis Rhed. Front. Plant Sci. 12:650485. doi: 10.3389/fpls.2021.650485

Maathuis, F. J. M. (2014). Sodium in plants: perception, signalling, and regulation of sodium fluxes. J. Exp. Bot. 65, 849-858. doi: 10.1093/jxb/ert326
Marschner, P. (2012). Marschner's Mineral Nutrition of Higher Plants. 3rd Edn. London: Academic Press.

Pavlovic, J., Kostic, L., Bosnic, P., Kirkby, E. A., and Nikolic, M. (2021). Interactions of silicon with essential and beneficial elements in plants. Front. Plant Sci. 12:697592. doi: 10.3389/fpls.2021.69 7592

Pueyo, J. J., Quiñones, M. A., Coba de la Peña, T., Fedorova, E. E., and Lucas, M. M. (2021). Nitrogen and phosphorus interplay in lupin root nodules and cluster roots. Front. Plant Sci. 12:644218. doi: 10.3389/fpls.2021.644218

Tripathi, D. K., Singh, V. P., Lux, A., and Vaculik, M. (2020). Silicon in plant biology: from past to present, and future. J. Exp. Bot. 71, 6699-6702. doi: 10.1093/jxb/eraa448

Conflict of Interest: The authors declare that the research was conducted in the absence of any commercial or financial relationships that could be construed as a potential conflict of interest.

Publisher's Note: All claims expressed in this article are solely those of the authors and do not necessarily represent those of their affiliated organizations, or those of the publisher, the editors and the reviewers. Any product that may be evaluated in this article, or claim that may be made by its manufacturer, is not guaranteed or endorsed by the publisher.

Copyright (c) 2021 Romera, Lan, Rodríguez-Celma and Pérez-Vicente. This is an open-access article distributed under the terms of the Creative Commons Attribution License (CC BY). The use, distribution or reproduction in other forums is permitted, provided the original author(s) and the copyright owner(s) are credited and that the original publication in this journal is cited, in accordance with accepted academic practice. No use, distribution or reproduction is permitted which does not comply with these terms. 